\title{
BOUNDEDLY HOLOMORPHIC CONVEX RIEMANN DOMAIN
}

\author{
DONG S. KIM
}

\begin{abstract}
A boundedly holomorphic convex Riemann domain with a bounded spread map is a Stein manifold of bounded type.
\end{abstract}

In [2], we have defined a boundedly holomorphic convex domain as a holomorphic convex domain determined by bounded holomorphic functions and a Stein manifold of bounded type as a Stein manifold defined by global bounded holomorphic functions in the place of global holomorphic functions in its definition. We denote $B(D)$ the algebra of bounded holomorphic functions on $D$.

Lemma. Let $(X, A: \alpha)$ be a Riemann domain with a bounded spread map $\alpha$;

$$
\alpha=\left(f_{1}, \cdots, f_{n}\right), \quad f_{i} \in B(X), 1 \leqq i \leqq n .
$$

Let $R$ be an equivalence relation $x \sim y$ if and only if $f(x)=f(y)$ for all $f \in B(X)$. Then the quotient space $E=X \mid R$ is a Riemann domain, $B(E)$ separates points on $E$, and $B(E)=B(X)$. Furthermore, if $X$ is boundedly holomorphic convex then the canonical map $\pi: X \rightarrow E=X / R$ is proper.

Proof. It is clear that $E$ is Hausdorff with the quotient topology. $\pi$ is locally one-to-one; for an open set $U$ where $\alpha$ is a homeomorphism, $\pi \mid U$ is one-one, for, if $x \neq y, x, y \in U$ then $\alpha(x) \neq \alpha(y)$; thus $f_{j}(x) \neq f_{j}(y)$ for some $j, 1 \leqq j \leqq n$. Thus $x \nsim y$. To show $\pi$ is open we shall show that, for a sufficiently small open subset $U$ in $X, \pi^{-1}(\pi U)$ is open in $X$. Let $P$ be a polydisc in $X$ so that $\alpha \mid P$ is a homeomorphism and $\pi \mid P$ is one-one. Take $x \in \pi^{-1}(\pi P)$; then $\pi(x) \in \pi P$. Thus there exists $y \in P$ such that $\pi(x)=\pi(y)$. So $f(x)=f(y)$ for all $f \in B(X)$, in particular, $f_{i}(x)=f_{i}(y)$ for $1 \leqq i \leqq n$. Hence $\alpha(x)=\alpha(y)$. Take $P_{x}, P_{y}$ in $U$; two polydiscs with centers $x$ and $y$ with the same radius. Note that $\alpha P_{x}=\alpha P_{y}$; a polydisc in $C^{n}$ of center $\alpha(x)=\alpha(y)$. For $v \in P_{x}$, put $w=\left(\alpha \mid P_{y}\right)^{-1}(\alpha(v)) \in P_{y}$ such that $\alpha(v)=\alpha(w)$,

Received by the editors December 1, 1972.

AMS (MOS) subject classifications (1970). Primary 32E05, 32E10.

Key words and phrases. Boundedly holomorphic convex domain, Riemann domain, Stein manifold of bounded type.

(c) American Mathematical Society 1973 
then the power series at $\alpha(x)$ is

$$
\begin{aligned}
f(v) & =\sum\left(j_{1} ! \cdots j_{n} !\right)^{-1} \frac{\partial_{\alpha}^{j_{1}+\cdots+j_{n}}}{\partial z_{1}^{j_{1}} \cdots \partial z_{n}^{j_{n}}} f(x)\left\{\alpha(v)_{1}-\alpha(x)_{1}\right\}^{j_{1}} \cdots\left\{\alpha(v)_{n}-\alpha(x)_{n}\right\}^{j_{n}} \\
& =\sum\left(j_{1} ! \cdots j_{n} !\right)^{-1} \frac{\partial_{\alpha}^{j_{1}+\cdots}+j_{n}}{\partial z_{1}^{j_{1}} \cdots \partial z_{n}^{j_{n}}} f(y)\left\{\alpha(w)_{1}-\alpha(x)_{1}\right\}^{j_{1}} \cdots\left\{\alpha(w)_{n}-\alpha(x)_{n}\right\}^{j_{n}} \\
& =f(w), \quad \text { where } \frac{\partial \alpha}{\partial z_{j}} f(x)=\frac{\partial}{\partial z_{j}} f \circ\left(\alpha \mid P_{x}\right)^{-1}(\alpha(x)), \quad 1 \leqq j \leqq n .
\end{aligned}
$$

Hence $f(v)=f(w)$ for all $f \in B(X)$. Thus, for every $v \in P_{x}$, there exists $w \in P_{y} \subset U$ such that $\pi(v)=\pi(w)$, and so $P_{x} \subset \pi^{-1}(\pi U)$. Therefore $\pi^{-1}(\pi U)$ is open in $X$. Since $\pi^{-1}(\pi U)$ is open in $X, \pi U$ is open in $E$. So $\pi: X \rightarrow E$ is a local homeomorphism.

Now, for $\tilde{x} \in E$, define $\beta(\tilde{x})=\alpha(x)$, where $\pi x=\tilde{x}$. Then $\beta: E \rightarrow C^{n}$ is a spread map. Moreover, since $\tilde{f}(\tilde{x})=f(x)$ for $\pi x=\tilde{x}, \tilde{f} \in B(E)$, and $f \in B(X), B(X)=B(E)$.

Finally, we show that $\pi$ is proper. For a compact subset $L$ in $E$ there is a compact subset $K$ in $X$ such that $\pi(K)=L$; for every point $\tilde{x} \in L$ there is a compact neighborhood $V_{x}$ of $\pi^{-1}(\tilde{x})$ in $X$ so that $\pi\left(V_{x}\right)$ is a compact neighborhood of $\tilde{x}$. Then there is a finite covering $\bigcup_{i}^{n} \pi\left(V_{x_{i}}\right) \supset L$ and $K_{1}=\bigcup_{i}^{n} V_{x_{i}}$ is compact. So $L \subset \pi\left(K_{1}\right)$. Hence $K=K_{1} \cap \pi^{-1}(L)$ is compact and $\pi(K)=L$. Now, let $\hat{K}=\left\{x \in X:|f(x)| \leqq\|f\|_{k}\right.$ for all $\left.f \in B(X)\right\}$, then $\hat{K} \supset \pi^{-1}(L)$, and so $\pi^{-1}(L)$ is compact. We complete the proof.

THEOREM. A boundedly holomorphic convex Riemann domain with a bounded spread map is a Stein manifold of bounded type.

Proof. Let $(X, A: \alpha)$ be a Riemann domain with a bounded spread map $\alpha$. Then by the Lemma we have a Riemann domain $E=X \mid R$ and a proper spread map $\pi: X \rightarrow E$. For a compact subset $L$ of $E$, set $L=\{\tilde{x} \in E$ : $|\tilde{f}(\tilde{x})| \leqq\|\tilde{f}\|_{L}$ for all $\left.\tilde{f} \in B(E)\right\}$. Since $\tilde{f}(\tilde{x})=\tilde{f}(\pi(x))=f(x),\|\tilde{f}\|_{L}=\|f\|_{\pi^{-1}(L)}$ and $\pi\left(\left\{\pi^{-1}(L)\right\}^{\wedge}\right)=\hat{L}$. Since $\pi$ is proper, $\pi^{-1}(L)$ is compact and so are $\left\{\pi^{-1}(L)\right\}^{\wedge}$ and $\pi\left(\left\{\pi^{-1}(L)\right\}^{\wedge}\right)$. Hence $E$ is boundedly holomorphic convex. Thus $E$ is a Stein manifold of bounded type. Therefore it suffices to show that $B(X)$ separates points of $X$. Since $\pi$ is a proper spread map, $\pi^{-1}(x)$ is finite for each $\tilde{x} \in E$. Define a sheaf $\tilde{A}$ on $E$ by

$$
\tilde{A}_{\tilde{x}}=\sum_{x_{i} \in \pi^{-1}(\tilde{x})} \bigoplus A_{x_{i}}
$$

Then $\tilde{A}$ is a coherent sheaf on $E$ and bounded global sections on $X$ coincide with bounded global sections on $E$. Let $\pi^{-1}(x)=\left\{x_{1}, \cdots, x_{n}\right\}$. For a small open neighborhood $U$ of $\tilde{x}$ in $E$, there is a section $\varphi$ on $U\left(\varphi_{u}=\tilde{f}\right.$ for $\left.u \in U\right)$ whose $x_{i}$ and $x_{j}$ components are different for $i \neq j$. 
Since $E$ is a Stein manifold of bounded type, in particular, a Stein manifold, by Cartan's Theorem A, there is a global section $\Phi$ on $E$ such that $\Phi \mid U=\varphi$. Then the function $F \in \tilde{A}(E)$ determined by $\Phi$ separates the points $\left\{x_{1}, \cdots\right.$, $\left.x_{n}\right\}$. Now, since $E$ is a Stein manifold of bounded type, $F$ can be approximated by bounded functions in $B(E)=B(X)$ (see a note after Definition 3 in [2]). Hence there is a bounded holomorphic function on $X$ which separates the points $\left\{x_{1}, \cdots, x_{n}\right\}$, so that $n=1$. We complete the proof.

Since it has been known that a domain of bounded holomorphy need not be a boundedly holomorphic convex domain we give the following.

Proposition. Let $(X, A)$ be an analytic space and let $\left\{D_{n}\right\}$ be an infinite sequence of bounded holomorphic convex -domains. If $D=\bigcap_{n} D_{n}$ is open then $D$ is also a boundedly holomorphic convex domain.

Remark. It has been known that, for two domains $D_{1}$ and $D_{2}$ in $C$ which are domains of bounded holomorphy, $B\left(D_{1}\right)$ is algebraically isomorphic to $B\left(D_{2}\right)$ if and only if $D_{1}$ and $D_{2}$ are conformally equivalent. This is also true for higher dimensions as follows:

If $D_{1}$ and $D_{2}$ are Stein-Riemann domains of bounded type with bounded spread maps then $B\left(D_{1}\right)$ is algebraically isomorphic to $B\left(D_{2}\right)$ if and only if $D_{1}$ and $D_{2}$ are biholomorphic. This follows from the fact that, for such a domain $D$, the spectrum of $B(D)$ ( $B$ with c.o. topology) which is given by point evaluations is the envelope of bounded holomorphy (see [2]).

\section{REFERENCES}

1. R. C. Gunning and H. Rossi; Analytic functions of several complex variables, Prentice-Hall Series in Modern Analysis, Prentice-Hall, Englewood Cliffs, N.J., 1965. MR 31 \#4927.

2. D. S. Kim, Boundedly holomorphic convex domains, Pacific J. Math. (to appear).

3. F. Quigley, Lectures on several complex variables, Tulane University, New Orleans, La., 1964-1966. 32601

Department of Mathematics, University of Florida, Gainesville, Florida 